Pacific Journal of Mathematic 


\title{
REGULAR AND SEMISIMPLE MODULES
}

\author{
T. J. Cheatham AND J. R. Smith
}

A module is regular if all its submodules are (Cohn) pure. The family of all regular modules is closed under products if and only if $R / J(R)$ is a von Neumann regular ring. If each regular $R$-module is semisimple then $R$ is a $T$-ring. An extra condition is needed for the converse. Character modules and extensions of regular and semisimple modules are investigated.

1. Introduction. Rings will be associative with identity and modules will be (left) unitary. $R$ will denote a ring which is not assumed commutative unless specifically stated and $J(R)$ will denote the Jacobson radical of $R$. Fieldhouse [5] calls a module $B$ regular if each submodule $A$ of $B$ is pure in $B$, i.e., the inclusion $0 \rightarrow A \rightarrow B$ remains exact upon tensoring by any (right) $R$-module. Regular modules have been studied under different definitions by Ware [12], Zelmanowitz [14], and Ramamurthi and Rangaswamy [9]. A module is semisimple if it is a sum of simple modules. For a subset $A$ of a module $B,(0: A)$ will denote the left ideal $\{r \in R \mid r x=0$ for all $x \in A\}$.

2. Products. The class of all semisimple modules is closed under products if and only if $R / J(R)$ is a semisimple (Artinian) ring. This follows from the canonical embedding $R / J(R) \subseteq \Pi R / M$, where the product is taken over the set of maximal left ideals $M$ of $R$.

Lemma 1. If $I$ is a two-sided ideal of $R$, then $R / I$ is a regular ring if and only if $R / I$ is a regular left (or right) $R$-module.

Proof. For any left $R / I$-module $B$ and any right $R$-module $C$ we have canonical group isomorphisms:

$$
C \otimes_{R} B \simeq(C / C I) \otimes_{R} B \simeq(C / C I) \otimes_{R / I} B .
$$

-If follows that an $R / I$-module is regular as an $R / I$-module if and and only if it is regular as an $R$-module. This proves the lemma.

Corollary. If $R$ is a commutative ring, an $R$-module $B$ is regular if and only if $R /(0: x)$ is a regular ring for each $0 \neq x \in B$.

THEOREM 1. The following statements are equivalent for a ring $R$. 
(1) The family of all regular left $R$-modules is closed under products.

(2) The product of any family of semisimple left $R$-modules is regular.

(3) $R / J(R)$ is a regular ring.

(4) $A$ left $R$-module $B$ is regular if and only if $J(R) \cdot B=0$.

Proof. (1) $\rightarrow(2)$. A semisimple module is regular.

$(2) \rightarrow(3)$. Use Lemma 1 and the embedding $R / J(R) \hookrightarrow \Pi R / M$ where the product is taken over the set of all maximal left ideals $M$ of $R$.

$(3) \rightarrow(4)$. From Fieldhouse, [6, Theorem 2.3, pg. 194], $J(R) \cdot B=0$ for all regular left $R$-modules $B$. If $B$ is an $R$-module such that $J(R) \cdot B=0$, then $B$ is an $R / J(R)$-module. From (3), $B$ is a regular $R / J(R)$-module hence a regular $R$-module by the proof of Lemma 1 .

$(4) \rightarrow(1)$. If $\left\{B_{i} \mid i \in I\right\}$ is a family of left $R$-modules such that $J(R) \cdot B_{i}=0$ for each $i \in I$, then $J(R) \cdot\left(\Pi B_{i}\right)=0$.

Thus if each $B_{i}$ is regular so is $\Pi B_{i}$.

We note that in the commutative case each of (1)-(4) of Theorem 1 is equivalent to:

(5) An ideal $K$ of $R$ is an intersection of maximal ideals of $R$ if and only if $R / K$ is a regular ring.

In regard to (4) of Theorem 1 the following seems worth noting.

Proposition 1. Let $R$ be a commutative ring. Each nonzero regular $R$-module $B$ has a maximal submodule. Moreover, $J(B)=0$, where $J(B)$ is the radical of $B$.

Proof. Let $0 \neq x \in B$. By Zorn's lemma there is a submodule $Y$ of $B$ maximal in the collection of submodules $X$ of $B$ such that $x \notin X$. We show $Y$ is a maximal submodule of $B$. The submodule $D=\cap\{A \subseteq B \mid Y \varsubsetneqq A\}$ of $B$ contains $x$ so $D / Y \neq 0$. $D / Y$ is simple. Thus $D / Y$ is a direct summand of $B / Y$. (This will be shown in $\S 3$, Corollary 5.) There is a submodule $C$ of $B$ such that $Y \subseteq C, C \cap$ $D=Y$, and $B / Y=D / Y \oplus C / Y$. Since $x \notin Y, x \notin C$ so $Y=C$. Thus $B / Y=D / Y$ is simple. Also, $x \notin J(B)$ since $x \notin Y$. Hence $J(B)=0$.

This can be viewed as a generalization of the well-known fact: Over a commutative regular ring each module has a maximal submodule.

3. Character modules. Throughout this section $R$ will denote a commutative ring. By the character module of an $R$-module $E$ we mean the $R$-module $E^{+}=\operatorname{Hom}_{Z}(E, Q / Z)$ where $Q$ denotes the field of rational numbers and $Z$ denotes the ring of integers. The 
notion of a character module has been exploited by several authors; for example, Lambek [8], Enochs [4], Würfel [13].

THEOREM 2. Let $R$ be a commutative ring.

(1) For an $R$-module $E$, if $E^{+}$is regular so is $E$.

(2) The converse holds if and only if $R / J(R)$ is a regular ring.

Proof of (1). Let $0 \rightarrow C \rightarrow E \rightarrow D \rightarrow 0$ be an exact sequence of $R$-modules. Then $0 \rightarrow D^{+} \rightarrow E^{+} \rightarrow C^{+} \rightarrow 0$ is a pure exact sequence. Since $D^{+}$is pure-injective [11, Prop. 9.2] and pure in $E^{+}$(by our assumption), $0 \rightarrow D^{+} \rightarrow E^{+} \rightarrow C^{+} \rightarrow 0$ is split exact. It follows that $0 \rightarrow C \rightarrow E \rightarrow D \rightarrow 0$ is pure [11, Prop. 9.1]. Thus $E$ is regular.

Proof of (2). Assume $R / J(R)$ is a regular ring. We first note that $(0: E)=\left(0: E^{+}\right)$for any $R$-module $E$. For a regular $R$-module $E, J(R) \cdot E=0$ (by Proposition 1 ) so $J(R) \cdot E^{+}=0$. By Theorem $1 E^{+}$ is a regular $R$-module. Now assume the character module of each regular module is regular. Then $(\Sigma S)^{+} \simeq \Pi S^{+}$is regular (where the sum is taken over one copy of each simple module $S$ ). Since $(0: S)=\left(0: S^{+}\right), S^{+}$contains an isomorphic copy of $S$. Hence, we have an embedding $\Pi S \hookrightarrow \Pi S^{+}$. But $R / J(R) \hookrightarrow \Pi S$ so $R / J(R)$ is a regular $R$-module and a regular ring by Lemma 1 .

THEOREM 3. Let $R$ be a commutative ring. For an $R$-module $E, E^{+}$is semisimple if and only if $R /(0: E)$ is a semisimple (Artinian) ring.

Proof. Assume $R /(0: E)$ is a semisimple ring. Since $(0: E)=$ $\left(0: E^{+}\right), E^{+}$is a module over $R /(0: E)$. Hence $E^{+}$is semisimple.

Conversely, assume $E^{+}$is a semisimple module. We show $E$ is semisimple and $(0: E)$ is an intersection of a finite number of maximal ideals (hence $R /(0: E)$ is a semisimple ring). By Theorem $2 E$ is regular. Let $0 \neq x \in E$, and $R x \simeq R / I$ for some ideal $I$ of $R$. $R / I$ is a regular ring, and the exact sequence of $R$-modules $0 \rightarrow R / I \rightarrow E$ gives an exact sequence $E^{+} \rightarrow(R / I)^{+} \rightarrow 0$. Hence $(R / I)^{+}$is semisimple. We must show $R / I$ is semisimple. Thus we may reduce to the case where $R$ is a regular ring and $R^{+}$is a semisimple $R$-module. We show $R$ is semisimple.

Let $\left\{e_{k} \mid k \in K\right\}$ be any set of orthogonal idempotents in $R$. For each $k \in K$ choose a maximal ideal $M_{k}$ of $R$ such that $M_{k}=N_{k} \oplus$ $R\left(1-e_{k}\right)$ where $N_{k}$ is a maximal ideal of $\operatorname{Re}_{k}$. There is an $R$ epimorphism $\mathrm{Re}_{k} \rightarrow R / M_{k}$ so an embedding $\left(R / M_{k}\right)^{+} \hookrightarrow\left(\mathrm{Re}_{k}\right)^{+}$. Also, $\Sigma \mathrm{Re}_{k} \leqq R$ so there is an $R$-epimorphism $R^{+} \rightarrow \Pi\left(\mathrm{Re}_{k}\right)^{+}$. Hence $\Pi\left(\mathrm{Re}_{k}\right)^{+}$is semisimple. We have $\Pi\left(R / M_{k}\right)^{+} \hookrightarrow \Pi\left(\mathrm{Re}_{k}\right)^{+}$so $\Pi\left(R / M_{k}\right)^{+}$ 
is semisimple. Now pick $y=\left(y_{k}\right) \in \Pi\left(R / M_{k}\right)^{+}$such that $y_{k} \neq 0$ for all $k$. Then $\left(0: y_{k}\right)=M_{k}$ for each $k \in K$. But $R y$ is semisimple so $(0: y)=H_{1} \cap \cdots \cap H_{m}$ where each $H_{i}$ is a maximal ideal of $R$. Since $(0: y) \cong\left(0: y_{k}\right), \prod_{\imath=1}^{m} H_{i} \cong M_{k}$ for each $k \in K$. It follows that $K$ is finite. So $R$ has only a finite number of orthogonal idempotents. Thus $R$ is semisimple as desired. We have shown: If $E^{+}$is a semisimple $R$-module so is $E$. Since $E$ is semisimple $(0: E)$ is an intersection of maximal ideals of $R$. Clearly, $R /(0: E)$ is a semisimple ring if and only if $(0: E)$ is an intersection of a finite number of maximal ideals (equivalently, only a finite number of simples are represented in $E$ ). Let $\left\{S_{k} \mid k \in K\right\}$ be a complete set of nonisomorphic simple submodules of $E$. The exact sequence $0 \rightarrow \Sigma S_{k} \rightarrow E$ yeilds an exact sequence $E^{+} \rightarrow \Pi S_{k}^{+} \rightarrow 0$ and hence $\Pi S_{k}^{+}$is a semisimple module. Now pick $y=\left(y_{k}\right) \in \Pi S_{k}^{+}$as above and proceed to show $K$ is finite.

CoRollary 1. Let $M_{1}, \cdots, M_{t}$ be distince maximal ideals of a commutative ring $R$ and for each $i=1,2, \cdots, t$ let $V_{i}$ be an $R / M_{i^{-}}$ vector space. Then $\left(\sum_{i=1}^{t} V_{i}\right)^{+}$is semisimple.

CoROLlary 2. If $S$ is a simple module over a commutative ring $R, S^{+}$is semisimple and consists of copies of $S$.

CoROLlary 3 (Kaplansky). Over a commutative ring $R$ a simple module $S$ is flat if and only if it is injective.

Proof. Assume $S$ is simple and flat. By Lambeck's result [8] $S^{+}$is injective. From Corollary $2 S$ is injective. Assume now $S$ is simple and injective. From Corollary 2 we have $S^{+}$is absolutely pure. Since $S^{+}$is pure-injective it is injective. Lambek's result shows $S$ is flat.

COROllary 4. Over a commutative ring $R$ a simple module is pure-injective.

COROLLARY 5. Over a commutative ring $R$ a simple submodule of a regular module is a direct summand.

An example of Faith [7], pg. 130 shows that Corollary 5 may not hold for a noncommutative ring.

4. Regular implies semisimple. Over any $\operatorname{ring} R$ a semisimple module is regular. It is known (see Cheatham [2] or Fieldhouse [6]) that over a (not necessarily commutative) local ring each regular 
module is semisimple. Cheatham [2] proves that over a Noetherian ring each regular module is semisimple. This latter result can be weakened slightly to

Proposition 2. If $R$ is a ring such that all maximal left ideals of $R$ are finitely generated, then each regular left $R$-module is semisimple.

Proof. Let $0 \neq E$ be a regular left $R$-module, and $0 \neq x \in E$. We show $R x$ is semisimple. If $R x$ has a nonzero proper submodule it has a maximal such submodule $L$. There is a maximal ideal $M$ of $R$ such that $R x / L \simeq R / M$. We have a commutative diagram:

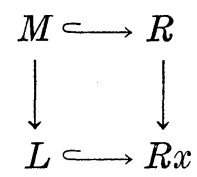

where the vertical maps are given by $r \mapsto r x$. Since $L$ is pure in $R x$ and $M$ is finitely generated there exists a homomorphism $f: R \rightarrow$ $L$ making the top triangle (in the resulting diagram) commutative. Let $f(1)=y \in L$. It can be shown that $R(y-x) \simeq R / M$ and $R(y-x) \oplus$ $L=R x$. Thus each maximal submodule of $R x$ is a direct summand of $R x$. It follows that $R x$ (hence $E$ ) is semisimple.

There is much work to be done in determining which noncommutative rings have all their regular modules semisimple. Before giving our results in the commutative case we require some definitions.

A module is called torsion if each nonzero factor module has a simple submodule. A module $E$ is called S-primary for a simple module $S$ if each nonzero factor module of $E$ has a simple submodule isomorphic to $S$. Dickson [3] calls a ring $R$ a $T$-ring if each torsion module decomposes into a direct sum of its primary components. Shores [10] calls a ring $R$ a special ring if $R$ is a non-Noetherian semiprime ring whose socle is a maximal ideal.

Lemma 3. Let $R$ be a commutative ring, and $S$ a simple $R$ module. The S-primary component $E_{S}$ of a regular module $E$ is semisimple and is a direct summand of $E$.

Proof. Let $M$ be an ideal in $R$ such that $S \simeq R / M$. The socle of $E_{S}$, Soc $\left(E_{S}\right)$, is a pure-injective $R / M$-module hence a pure-injective $R$-module [1, pg. 29]. Thus $E=\operatorname{Soc}\left(E_{S}\right) \oplus T$ for some submodule $T$ of $E$. But Soc $\left(E_{S}\right)$ is large in $E_{S}$ so $E_{S}=\operatorname{Soc}\left(E_{S}\right)$ and the lemma is proved. 
COROLLARY. An S-primary regular module is semisimple.

Examples of regular torsion modules which are not semisimple abound. Such an example exists over any ring which is not a $T$ ring as shown by the following lemma:

Lemma 4. A commutative ring $R$ is a T-ring if and only if each regular torsion module is semisimple.

Proof. A torsion module over a T-ring decomposes into a sum of its primary components. By the last corollary each primary component of a regular torsion module is semisimple.

Conversely, if $R$ is not a T-ring, $R$ has a special factor ring $R / I$ (Shores [10]). $R_{0} / I$ is a regular ring so a regular $R_{0}$-module by Lemma $1 . \quad R_{0} / I$ is also a torsion $R_{0}$-module but is not semisimple.

THEOREM 4. Let $R$ denote a commutative ring. The following statements are equivalent.

(1) Each regular $R$-module is semisimple.

(2) An $R$-module $E$ is semisimple if and only if $E_{M}$ is a semisimple $R_{M}$-module for each maximal ideal $M$ of $R$.

(3) (a) $R$ is a T-ring.

(b) Each regular $R$-module is torsion.

Proof. The equivalence of (1) and (2) was noted in [2]. It is a consequence of the fact that "regular implies semisimple" over a local ring and that (2) holds with "semisimple" replaced by "regular". $(1) \rightarrow(3 a)$. A regular factor ring of $R$ must be semisimple by (1). Thus there is no special factor ring of $R$ and $R$ is a $T$-ring.

$(1) \rightarrow(3 b)$. A semisimple module is torsion.

$(3) \rightarrow(1)$. This is an immediate consequence of Lemma 4 .

Neither (3a) nor (3b) is sufficient by itself to imply (1). Tom Shorem has shown (unpublished) that the product of an infinite number of fields (or an infinite number of copies of a single field) is a $T$-ring. Thus (3a) does not imply (1). The subring $R$ of $\prod_{i=1}^{\infty} F_{i}$ (where for each $i, F_{i} \cong F$, a field) generated by $\bigoplus_{i=1}^{\infty} F_{i}$ and the unit $e$ of $\prod_{i=1}^{\infty} F_{\imath}$ is not a $T$-ring (cf. Dickson [3]). In fact $R$ is a special ring. In this regard we note

Proposition 3. If $R$ is a commutative ring whose socle Soc $R$ is a maximal ideal of $R$, then each regular $R$-module is torsion.

Proof. It is sufficient to show that a cyclic regular module has a simple submodule. Let $I$ be an ideal in $R$ such that $0 \neq R / I$ is 
a regular module. If there is a minimal ideal $S$ of $R$ such that $S \nsubseteq I$ then $(S+I) / I$ is a simple submodule of $R / I$. Otherwise, Soc $R \subseteq I$ so Soc $R=I$ and $R / I$ is simple.

The ring of integers would serve as a counter-example to the converse of Proposition 3.

5. Extensions, Let $0 \rightarrow A \rightarrow B \rightarrow C \rightarrow 0$ be an exact sequence of $R$-modules. In this section we give necessary and sufficient conditions for $B$ to be regular (semisimple) whenever $A$ and $C$ are regular (semisimple).

THEOREM 5. The following statements are equivalent for a commutative ring $R$.

(1) Each torsion $R$-module is semisimple.

(2) $R$ is a T-ring and $M^{2}=M$ for all maximal ideals $M$ of $R$.

(3) If $0 \rightarrow A \rightarrow B \rightarrow C \rightarrow 0$ is exact with $A$ and $C$ semisimple then $B$ is semisimple.

Proof. (1) $\rightarrow(2)$. Semisimple modules obviously decompose into primary components so $R$ is a $T$-ring. We have an exact sequence of $R$-modules:

$$
0 \longrightarrow M / M^{2} \longrightarrow R / M^{2} \longrightarrow R / M \longrightarrow 0 \text {. }
$$

$M / M^{2}$ and $R_{0} / M$ are torsion (in fact, semisimple) so $R_{0} / M^{2}$ is torsion and hence semisimple. Thus $\left({ }^{*}\right)$ splits and $R / M^{2}$ is an $R / M$-module. Therefore $M / M^{2}=0$, i.e. $M=M^{2}$.

(2) $\rightarrow(3)$. Let $0 \rightarrow A \rightarrow B \rightarrow C \rightarrow 0$ be an exact sequence of $R$ modules with $A$ and $C$ semisimple. Then $B$ is torsion and, as $R$ is a $T$-ring, $B$ decomposes into its primary components $B_{S}$. For each maximal ideal $M$ of $R$ we have an exact sequence of primary components $0 \rightarrow A_{S} \rightarrow B_{S} \rightarrow C_{S} \rightarrow 0$ where $S \simeq R / M$. Since $A_{S}$ and $C_{S}$ are $R / M$-modules, $B_{S}$ is an $R / M^{2}$-module. But $M=M^{2}$ so $B_{S}$ is semisimple. Thus $B$ is semisimple.

$(3) \rightarrow(1)$ Let $E$ be a torsion module and consider the exact sequence

$$
0 \longrightarrow \operatorname{Soc}(E) \longrightarrow L_{1}(E) \longrightarrow \operatorname{Soc}(E / \operatorname{Soc}(E)) \longrightarrow 0
$$

where $L_{1}(E) \subseteq E$. By (3) $L_{1}(E)$ is semisimple so Soc $(E)=L_{1}(E)$. Consequently, $\operatorname{Soc}(E / \operatorname{Soc}(E))=0$. But $E$ is torsion so $E / \operatorname{Soc}(E)=0$ and $E=\operatorname{Soc}(E)$ as desired.

THEOREM 6. The following statements are equivalent for a commutative ring $R$.

(1) Each torsion $R$-module is regular. 
(2) $M=M^{2}$ for all maximal ideals $M$ of $R$.

(3) If $0 \rightarrow A \rightarrow B \rightarrow C \rightarrow 0$ is exact with $A$ and $C$ regular, then $B$ is regular.

(4) If $I$ and $K$ are ideals of $R$ such that $R / I$ and $R / K$ are regular $R$-modules then $R / I K$ is a regular $R$-module.

Proof. (1) $\rightarrow(2)$. For a maximal ideal $M$ of $R, R / M^{2}$ is a torsion $R$-module. By (1) $R / M^{2}$ is a regular $R$-module. We show $M / M^{2}=0$. Let $0 \neq a \in M$, by the purity of $M / M^{2}$ in $R / M^{2}$ we have $a\left(M / M^{2}\right)=$ $a\left(R / M^{2}\right) \cap\left(M / M^{2}\right)$. But $a \in M$, so $a\left(M / M^{2}\right)=0$. So $a+M^{2} \in a\left(R / M^{2} \cap\right.$ $\left(M / M^{2}\right)=0$, i.e., $a \in M^{2}$.

$(2) \rightarrow(3)$. Each localization $R_{M}$ of $R$ at a maximal ideal $M$ of $R$ satisfies (2). Clearly $R_{M}$ is a $T$-ring. An $R_{M}$-module is regular if and only if it is semisimple. Thus by Theorem 5 an extension of a regular $R_{M}$-module by a regular $R_{M}$-module is regular. Consider an exact sequence $0 \rightarrow A \rightarrow B \rightarrow C \rightarrow 0$ of $R$-modules with $A$ and $C$ regular. Localizing at a maximal ideal $M$ we obtain an exact sequence of $R_{M}$-modules $0 \rightarrow A_{M} \rightarrow B_{M} \rightarrow C_{M} \rightarrow 0$ with $A_{M}$ and $C_{M}$ regular $R_{M}$-modules. Thus $B_{M}$ is a regular $R_{M}$-module for all maximal ideals $M$ of $R$. This proves $B$ is a regular $R$-module.

$(3) \rightarrow(4)$. Let $I$ and $K$ be ideals of $R$ such that $R / I$ and $R / K$ are regular $R$-modules. $K / I K$ is a (regular) $R / I$-module so a regular $R$-module. We have an exact sequence of $R$-modules: $0 \rightarrow K / I K \rightarrow$ $R / I K \rightarrow R / K \rightarrow 0$. From (3) we get $R / I K$ is regular.

$(4) \rightarrow(1)$. Let $0 \neq E$ be a torsion $R$-module. We show that $E$ is locally a regular module. It will follow that $E$ is a regular $R$ module. We assume $E$ is cyclic. From (4), for each maximal ideal $M$ of $R$, we have $R / M^{2}$ is regular so $M=M^{2}$ as in the proof of $(1) \rightarrow(2)$. The local ring $R_{M}$ enjoys the same property. Thus each torsion $R_{M}$-module is semisimple (Theorem 5). The localization $E_{M}$ of $E$ at a maximal ideal $M$ is a torsion $R_{M}$-module. Therefore $E_{M}$ is a regular $R_{M}$-module as required.

The authors wish to express deep appreciation to Professor Edgar Enochs for his many helpful suggestions and to the University of Kentucky for the use of its facilities.

\section{REFERENCES}

1. D. D. Berry, S-purity, Ph. D. Dissertation, Uuiversity of Kentucky, (1975)

2. F. D. Cheatham, F-absolutely pure modules, Ph. D. Dissertation, University of Kentucky (1972)

3. S. E. Dickson, Decompositions of modules II, Math. Zeit., 104 (1968), 349-357.

4. E. E. Enochs, $A$ note on semihereditary rings, Canad Math. Bull., 16 (1973) 439-440.

5. D. J. Fieldhouse, Purity and flatness, Ph. D. Thesis, McGill University (1967). 
6. D. J. Fieldhouse, Regular modules over semi-local rings, Colloq. Math. Soc. Janos Bolyoi (1971).

7. C. Faith, Lectures on injective modules and quotient rings, Springer-Verlag, Berlin (1967).

8. J. Lambek, A module is flat if and only if its character module is injective, Canad. Math. Bull., 7 (1964), 237-243.

9. V. S. Ramamurthi and K. M. Rangaswamy, On finitely injective modules, J. Austral. Math. Soc., 16 (1973), 239-248.

10. T. S. Shores, Decompositions of finitely generated modules, Proc. Amer. Math. Soc., 30 (1971), 445-450.

11. B. T. Stenström, Pure submodules, Arch. Math., Band $7 \mathrm{nr}$ 10, (1967), 158-171.

12. R. Ware, Endomorphism rings of projective modules, Trans. Amer. Math. Soc., 155 (1971), 233-256.

13. T. Würfel, Uber absolut reine ringe, J. Reine Angew. Math., 262-263 (1973), 381-391.

14. J. Zelmanowitz, Regular modules, Trans. Amer. Math. Soc., 163 (1972), 341-355.

Received March 18, 1974. The first named author was supported by Samford University Research Fund grant \#51.

SAMFORD UNIVERSITY 



\section{PACIFIC JOURNAL OF MATHEMATICS}

\section{EDITORS}

RICHARD ARENS (Managing Editor)

University of California

Los Angeles, California 90024

\section{R. A. Beaumont}

University of Washington

Seattle, Washington 98105
J. DugundjI

Department of Mathematics

University of Southern California

Los Angeles, California 90007

D. Gilbarg and J. Milgram

Stanford University

Stanford, California 94305

\section{ASSOCIATE EDITORS}
E. F. BECKENBACH
B. H. NeumanN
F. WOLF
K. YosHIDA

\section{SUPPORTING INSTITUTIONS}

\author{
UNIVERSITY OF BRITISH COLUMBIA \\ CALIFORNIA INSTITUTE OF TECHNOLOGY \\ UNIVERSITY OF CALIFORNIA \\ MONTANA STATE UNIVERSITY \\ UNIVERSITY OF NEVADA \\ NEW MEXICO STATE UNIVERSITY \\ OREGON STATE UNIVERSITY \\ UNIVERSITY OF OREGON \\ OSAKA UNIVERSITY
}

\author{
UNIVERSITY OF SOUTHERN CALIFORNIA \\ STANFORD UNIVERSITY \\ UNIVERSITY OF HAWAII \\ UNIVERSITY OF TOKYO \\ UNIVERSITY OF UTAH \\ WASHINGTON STATE UNIVERSITY \\ UNIVERSITY OF WASHINGTON \\ AMERICAN MATHEMATICAL SOCIETY
}

The Supporting Institutions listed above contribute to the cost of publication of this Journal, but they are not owners or publishers and have no responsibility for its content or policies.

Mathematical papers intended for publication in the Pacific Journal of Mathematics should be in typed form or offset-reproduced, (not dittoed), double spaced with large margins. Please do not use built up fractions in the text of your manuscript. You may however, use them in the displayed equations. Underline Greek letters in red, German in green, and script in blue. The first paragraph or two must be capable of being used separately as a synopsis of the entire paper. Items of the bibliography should not be cited there unless absolutely necessary, in which case they must be identified by author and Journal, rather than by item number. Manuscripts, in triplicate, may be sent to any one of the editors. Please classify according to the scheme of Math. Reviews, Index to Vol. 39. All other communications should be addressed to the managing editor, or Elaine Barth, University of California, Los Angeles, California, 90024.

The Pacific Journal of Mathematics expects the author's institution to pay page charges, and reserves the right to delay publication for nonpayment of charges in case of financial emergency.

100 reprints are provided free for each article, only if page charges have been substantially paid. Additional copies may be obtained at cost in multiples of 50 .

The Pacific Journal of Mathematics is issued monthly as of January 1966. Regular subscription rate: $\$ 72.00$ a year (6 Vols., 12 issues). Special rate: $\$ 36.00$ a year to individual members of supporting institutions.

Subscriptions, orders for back numbers, and changes of address should be sent to Pacific Journal of Mathematics, 103 Highland Boulevard, Berkeley, California, 94708.

PUBLISHED BY PACIFIC JOURNAL OF MATHEMATICS, A NON-PROFIT CORPORATION

Printed at Kokusai Bunken Insatsusha (International Academic Printing Co., Ltd.), 8-8, 3-chome, Takadanobaba, Shinjuku-ku, Tokyo 160, Japan. 


\section{Pacific Journal of Mathematics \\ Vol. 65, No. $2 \quad$ October, 1976}

Andrew Adler, Weak homomorphisms and invariants: an example .......... 293

Howard Anton and William J. Pervin, Separation axioms and metric-like

functions ............................................. 299

Ron C. Blei, Sidon partitions and p-Sidon sets .................... 307

T. J. Cheatham and J. R. Smith, Regular and semisimple modules ........... 315

Charles Edward Cleaver, Packing spheres in Orlicz spaces .............. 325

Le Baron O. Ferguson and Michael D. Rusk, Korovkin sets for an operator on a

space of continuous functions ............................. 337

Rudolf Fritsch, An approximation theorem for maps into Kan fibrations ....... 347

David Sexton Gilliam, Geometry and the Radon-Nikodym theorem in strict

Mackey convergence spaces .................................

William Hery, Maximal ideals in algebras of topological algebra valued

functions ...................................... 365

Alan Hopenwasser, The radical of a reflexive operator algebra ........... 375

Bruno Kramm, A characterization of Riemann algebras................. 393

Peter K. F. Kuhfittig, Fixed points of locally contractive and nonexpansive

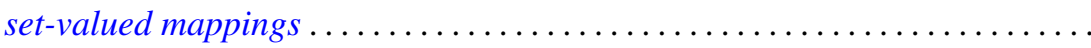

Stephen Allan McGrath, On almost everywhere convergence of Abel means of

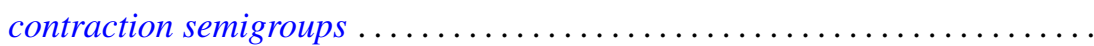

Edward Peter Merkes and Marion Wetzel, A geometric characterization of

indeterminate moment sequences............................ 409

John C. Morgan, II, The absolute Baire property ................... 421

Eli Aaron Passow and John A. Roulier, Negative theorems on generalized convex approximation .................................... 437

Louis Jackson Ratliff, Jr., A theorem on prime divisors of zero and characterizations of unmixed local domains ..............

Ellen Elizabeth Reed, A class of $T_{1}$-compactifications................... 471

Maxwell Alexander Rosenlicht, On Liouville's theory of elementary

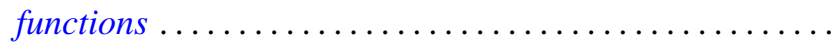

Arthur Argyle Sagle, Power-associative algebras and Riemannian

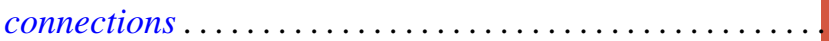

Chester Cornelius Seabury, On extending regular holomorphic maps from Stein manifolds...

Elias Sai Wan Shiu, Commutators and numerical ranges of powers of operators ...................................

Donald Mark Topkis, The structure of sublattices of the product of $n$ lattices ... 525

John Bason Wagoner, Delooping the continuous $K$-theory of a valuation

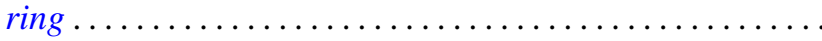

Ronson Joseph Warne, Standard regular semigroups...........

Anthony William Wickstead, The centraliser of $E \otimes_{\lambda} F \ldots$. 